\title{
Sensitivity Study of Borehole-to-Surface and Cross-Well Electromagnetic Measurements Acquired with Energized Steel Casing to Water Displacement in Hydrocarbon-Bearing Layers
}

\author{
David Pardo ${ }^{1}$, Carlos Torres-Verdin ${ }^{1}$, and (Ian) Zhiyi Zhang ${ }^{2}$
}

\begin{abstract}
We study the theoretical response of electromagnetically energized steel casing in the presence of subsurface variations of electrical resistivity. Casing is energized with a finite-size solenoid antenna located along the axis of the borehole. Measurements consist of the azimuthal component of the electric field acquired either on the surface or in a separate well in the same hydrocarbon field. We assume two-dimensional (2D) axisymmetric variations of subsurface electrical resistivity and casing excitation. Simulations of electromagnetic (EM) fields excited by energized steel casing are performed with a goal-oriented $h p$-adaptive finite-element method that automatically generates a sequence of optimal grids delivering exponential convergence rates in terms of the EM fields at the receiver antennas against CPU time. This advanced finite-element method enables accurate modeling of problems with high conductivity contrasts in large computational domains. Numerical simulations quantify the sensitivity of the measurements to variations of frequency, distance from casing to receivers, resistivity of the target oil-bearing layer, and pistonlike radial invasion of water within a target layer initially saturated with oil. When receivers are placed in a nearby well, numerical results indicate that measurements exhibit the largest sensitivity to the target (oil-saturated) layer when the transmitter or receiver antenna is located just above the target layer, and another antenna is located below the target layer. A frequency range from $5 \mathrm{~Hz}$ to $30 \mathrm{~Hz}$ provides optimal results for the detection of oil-bearing layers and estimation of radial extent of water invasion. Large horizontal distances (up to $1500 \mathrm{~m}$ ) between transmitter and receivers and a background material with resistivity above $50 \Omega \mathrm{m}$ also enhance the sensitivity of the measurements to radial variations of water invasion. This sensitivity may be as large as $15-20 \%$ of the measured electric field.
\end{abstract}

\section{INTRODUCTION}

This paper describes a model study to investigate the possibility of using steel casing as an electromagnetic (EM) source to detect and quantify oil-saturated formations and to estimate time-lapse variations of electrical resistivity due to fluid movement in an active hydrocarbon reservoir. Complementary experimental results in this area of research can be found in Kirkendall et al. (1999), Hoversten et al. (1995), and Bevc and Morrison (1991). In this work, we employ a numerical method to simulate cross-well and borehole-to-surface EM measurements in the presence of cased wells. In contrast to previous results, we avoid the use of semi-analytical approximations presented in Augustin et al. (1989) for a homo- 
geneous, isotropic, and unbounded formation. Simulations include the effect of arbitrary axial-symmetric distributions of formation conductivity. Although previous numerical simulations for cross-well and borehole-to-surface acquisitions exist for non-cased wells (Spies and Habashy, 1995; Sasaki et al., 1994), we develop similar results for steel-cased wells. We assume that casing contains no gaps. Thus, results differ from those presented by Nekut (1995), where he assumed a casing model with specifically designed insulated gaps. More recent work concerning energized steel casing can be found in Newmark et al. (1999), wherein an array of steel-casing segments is used as electrodes. In contrast to previous work, we present a detailed sensitivity analysis with respect to frequency, radial extent of water invasion within the target layer, and various locations of transmitter and receiver antennas.

In our model, we assume a vertical well penetrating axial-symmetric subsurface variations of electrical resistivity with respect to the axis of the well. The EM source used to excite current conduction in the casing is also assumed axial-symmetric with respect to the axis of the borehole. Casing is connected to an external, surface-controlled power source in the form of a contact electrode. This condition causes the electrical current to leak through the casing as well as through the subsurface rock formations in contact with the cased well. Thus, casing serves as a large EM source and the induced azimuthal component of the electric field can be simulated either on the surface or in a separate well located in the same hydrocarbon field. We also assume pistonlike invasion and consider inductive (solenoidal) sources operating at several frequencies in the range from 0.01 to $1000 \mathrm{~Hz}$.

The main objective of this paper is to study several variations of the subsurface model to quantify the sensitivity of the electric field to perturbations of the radial length of invasion within a target layer. We also study the influence of the location of the contact point of current along the steel-cased well on EM field measurements acquired 0-1500m radially away from the source. In addition, numerical sensitivity analyses are performed to quantify the influence of frequency and resistivity of the target (oil-saturated) layer on the EM fields measured either on the surface or in a separate well.

We quantify the sensitivity $S$ with respect to a model parameter (for example, water invasion) using the formula $S=\frac{\left|E_{b}-E_{p}\right|}{\left|E_{b}\right|}$, where $E_{p}$ indicates the electric field at the receiver for the background model (without water invasion), and $E_{p}$ is the electric field at the receiver for the perturbed model (including water invasion). Consistently, $S * 100$ indicates the sensitivity in percentage.

In all the simulations we employ a solenoidal coil and measure the azimuthal component of the electric field. When using different types of antennas (toroidal coil and a ring of vertical or horizontal electric dipoles) and/or measuring the vertical component of the magnetic field, numerical simulations indicated similar sensitivities with respect to water invasion. Thus, results corresponding to these cases have been omitted in the paper for brevity and clarity of the results.

Major challenges associated with the numerical simulations of these applications include the need to accurately resolve rapid spatial variations in the solution as a result of high conductivity contrasts, the need to consider large computational domains, the existence of dynamic ranges ${ }^{1}$ as large as $10^{14}$, and the need to quantify small relative differences in the measurements affected by radial variations of electrical conductivity due to water injection.

\footnotetext{
${ }^{1}$ quotient between the solution at the transmitter antenna and the EM fields at the receiver antenna
} 
All these challenges are solved using a numerical procedure based on a self-adaptive, goal-oriented $h p$-Finite Element Method (FEM) (Demkowicz, 2006; Pardo et al., 2006b) that converges exponentially in terms of the EM fields at the receiver antennas versus the problem size (as well as CPU time). This method generates a sequence of quasi-optimal $h p$-grids that incorporate local isotropic and anisotropic refinements in terms of both the element size, $h$, and the polynomial order of approximation, $p$. Continuity of the solution in the presence of such local refinements is ensured with the use of the hanging-node ${ }^{2}$ technology. The final $h p$-grid is intended to accurately approximate the solution in the quantity of interest (for example, a differential measurement of the EM fields). Our method for constructing the optimal grid is based on a purely numerical, highly accurate, and very reliable adaptive algorithm, which has been successfully applied to simulate a variety of resistivity logging measurements acquired with different devices, including through-casing (Pardo et al., 2006c), induction (Pardo et al., 2006a,c), and normal/laterolog instruments (Pardo et al., 2006b). This method has been validated against semi-analytical formulas (Pardo et al., 2006b; Paszynski et al., 2005), a radial code (Pardo et al., 2007b), and different 2D methods, such as a hybrid 2D mode-matching-finite-element method (Pardo et al., 2006c) and another finite-element software. This method is especially suited for the simulation of problems that involve steel casing, given the large exponential attenuation of the induced EM fields and the relatively large distance between the energized casing and the observation points. In contrast to existing finite-difference or integral-equation algorithms, the $h p$-adaptive methodology exhibits relative accuracy errors lower than $0.1 \%$ thereby enabling accurate and reliable sensitivity studies for a wide variety of perturbations of subsurface electrical resistivity, casing conductance, and frequency. Due to the extensive previous validation of the method used in this work, further validation is not presented in the paper. A detailed description of the simulation method can be found in Pardo et al. (2006a).

We first describe our model problem of interest. Then, we analyze the corresponding simulated measurements, with the conclusions presented thereafter. An Appendix is included to describe the variational formulation governing our EM logging applications of interest.

\section{NUMERICAL MODELS}

Assuming axial symmetry with respect the axis of the borehole, and with the surface located at $z=0 \mathrm{~m}$, we consider the following geometry, sources, receivers, and materials (illustrated in Figure 1):

- One $5 \mathrm{~cm}$-long and $2 \mathrm{~cm}$-thick solenoid antenna located in the inner-part of the casing, and $3 \mathrm{~m}$ below the surface.

- One $5 \mathrm{~cm}$-long and $2 \mathrm{~cm}$-thick receiving antenna located on a nearby well or $3 \mathrm{~m}$ below the surface. The horizontal position will be specified in each case, varying from $\rho=0 \mathrm{~m}$ up to $\rho=1500 \mathrm{~m}$, where $\rho$ designates the distance in the horizontal direction from the axis of symmetry.

- Borehole: a cylinder $\Upsilon_{I}$ of radius $13.97 \mathrm{~cm}$ surrounding the axis of symmetry $\left(\Upsilon_{I}=\right.$ $\{(\rho, \phi, z): \rho \leq 13.97 \mathrm{~cm}\})$, with resistivity $\mathrm{R}=1 \Omega \mathrm{m}$.

\footnotetext{
${ }^{2}$ Nodes with a solution value entirely dependent upon the solution values in neighboring nodes
} 
- Casing: a steel casing $\Upsilon_{I I}$ of width $1.27 \mathrm{~cm}$ surrounding the borehole $\left(\Upsilon_{I I}=\{(\rho, \phi, z)\right.$ : $13.97 \mathrm{~cm} \leq \rho \leq 15.24 \mathrm{~cm}\})$, with resistivity $\mathrm{R}=2.3 \times 10^{-7} \Omega \mathrm{m}$. The casing is 700 m long.

- Cement: a cement layer $\Upsilon_{I I I}$ of width $5.08 \mathrm{~cm}$ surrounding the casing $\left(\Upsilon_{I I I}=\right.$ $\{(\rho, \phi, z): 15.24 \mathrm{~cm} \leq \rho \leq 20.32 \mathrm{~cm}\})$, with resistivity $\mathrm{R}=2 \Omega \mathrm{m}$. The cement layer is $700 \mathrm{~m}$ long.

- Formation Material I (oil-saturated): a subdomain $\Upsilon_{I V}$ defined by $\Upsilon_{I V}=\{(\rho, \phi, z)$ : $\rho>20.32 \mathrm{~cm}, 475 \mathrm{~m} \leq z \leq 525 \mathrm{~m}\}$, with resistivity $\mathrm{R}=20 \Omega \mathrm{m}$.

- Formation Material II (rock): a subdomain $\Upsilon_{V}$ defined by $\Upsilon_{V}=\{(\rho, \phi, z): \rho>$ $20.32 \mathrm{~cm}, z<475 \mathrm{~m}$ or $z>525 \mathrm{~m}\}$, with resistivity $\mathrm{R}=5 \Omega \mathrm{m}$.

- Formation Material III (air): a subdomain $\Upsilon_{V I}$ defined by $\Upsilon_{V I}=\{(\rho, \phi, z): \rho>$ $20.32 \mathrm{~cm}, z>0 \mathrm{~m}\}$, with resistivity $\mathrm{R}=10^{6} \Omega \mathrm{m}$.

We will consider the case of pistonlike water injection into the oil-saturated layer (formation material I). The resulting water-saturated layer is assumed to have a resistivity equal to $5 \Omega \mathrm{m}$. We consider four different radii of water invasion: (a) No water invasion, (b) 10 $\mathrm{m}$ water invasion, (c) $100 \mathrm{~m}$ water invasion, and (d) $1000 \mathrm{~m}$ water invasion.

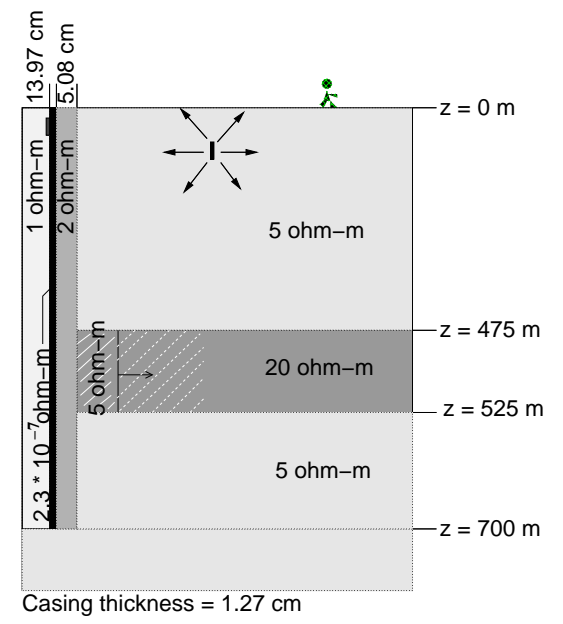

Figure 1: 2D cross section (radial distance versus vertical location) of the geometry assumed to simulate through-casing resistivity measurements. The model consists of one transmitter and one receiver antenna, a metallic casing, a cylindrical layer of cement, and three horizontal layers in the formation material with varying resistivities.

In the numerical experiments, we analyze the sensitivity of EM measurements to radial variations of water invasion in the hydrocarbon-bearing layer. We perform this sensitivity study as we vary the location of the transmitter and receiver antennas, the resistivity of the target (oil-saturated) layer, and the operating frequency (in a range from $0.01 \mathrm{~Hz}$ up to 1 $\mathrm{kHz})$. 


\section{Normalization and Frequency Dependence of Antennas}

EM conduction currents induced in both steel casing and subsurface rock formations originate from the use of inductive sources such as solenoids and toroids. The effective magnetic moment of these inductive devices depends not only on the circulating electrical current but also on the number of wire turns, cross-sectional area, perimeter, frequency of operation, and magnetic permeability of the core material (for the case of the toroid). Thus, actual values of EM fields for a given type of source can only be simulated if the pertinent geometrical and physical properties of the source are known beforehand. Simulations described in this paper consider unity electric moments and, therefore, need to be normalized with respect to actual device moments to yield the corresponding values of induced voltages. Simulation results described in this article have not been normalized to reflect measurement conditions. Such a step can only be performed if one is provided with the pertinent geometrical and physical properties of the measurement system.

\section{NUMERICAL RESULTS}

\section{Cross-Well Measurements. $5 \mathrm{~Hz}, 250 \mathrm{~m}$ (Horizontal Distance)}

Figure 2 displays the simulated measurements acquired in a nearby well located $250 \mathrm{~m}$ from the cased well. We assume an operating frequency equal to $5 \mathrm{~Hz}$. From the simulated measurements, we observe a sensitivity to radial extent of water invasion of up to $3 \%$ if the receiver is located below the target (oil-saturated) layer. For a receiver located above the target layer, the sensitivity to radial extent of water invasion rapidly decreases, and the intensity of the measured signal increases. For a pistonlike water invasion profile of radius below $200 \mathrm{~m}$, the sensitivity decreases to a level below $0.1 \%$. The phase of all simulated measurements is normalized to values between -180 and 180 degrees. Thus, the sudden variation occurring in the phase of the EM fields at $z=370 \mathrm{~m}$ is simply an effect of the selected normalization.

\section{Cross-Well Measurements. $30 \mathrm{~Hz}, 250 \mathrm{~m}$ (Horizontal Distance)}

Figure 3 displays the simulated measurements acquired in a nearby well located $250 \mathrm{~m}$ from the cased well. In this case, we assume an operating frequency equal to $30 \mathrm{~Hz}$. As we increase the frequency of operation up to $30 \mathrm{~Hz}$, we also observe an increase in the sensitivity with respect to radial extent of water invasion. Specifically, the sensitivity is as large as $10 \%$ when the receiver antennas are located below the target (oil-saturated) layer. We also observe that at $30 \mathrm{~Hz}$, the amplitude of the received signal is approximately 4 times smaller than at $5 \mathrm{~Hz}$.

\section{Cross-Well Measurements. $5 \mathrm{~Hz}, 900 \mathrm{~m}$ (Horizontal Distance)}

Figure 4 displays EM measurements simulated in a nearby well located $900 \mathrm{~m}$ from the cased well. We assume an operating frequency equal to $5 \mathrm{~Hz}$. From the simulated measurements, we observe a sensitivity to radial extent of water invasion of up to $5 \%$ if the receiver is located below the target (oil-saturated) layer. This sensitivity to water invasion is slightly 
X-well, $5 \mathrm{~Hz}$, horizontal distance $250 \mathrm{~m}$
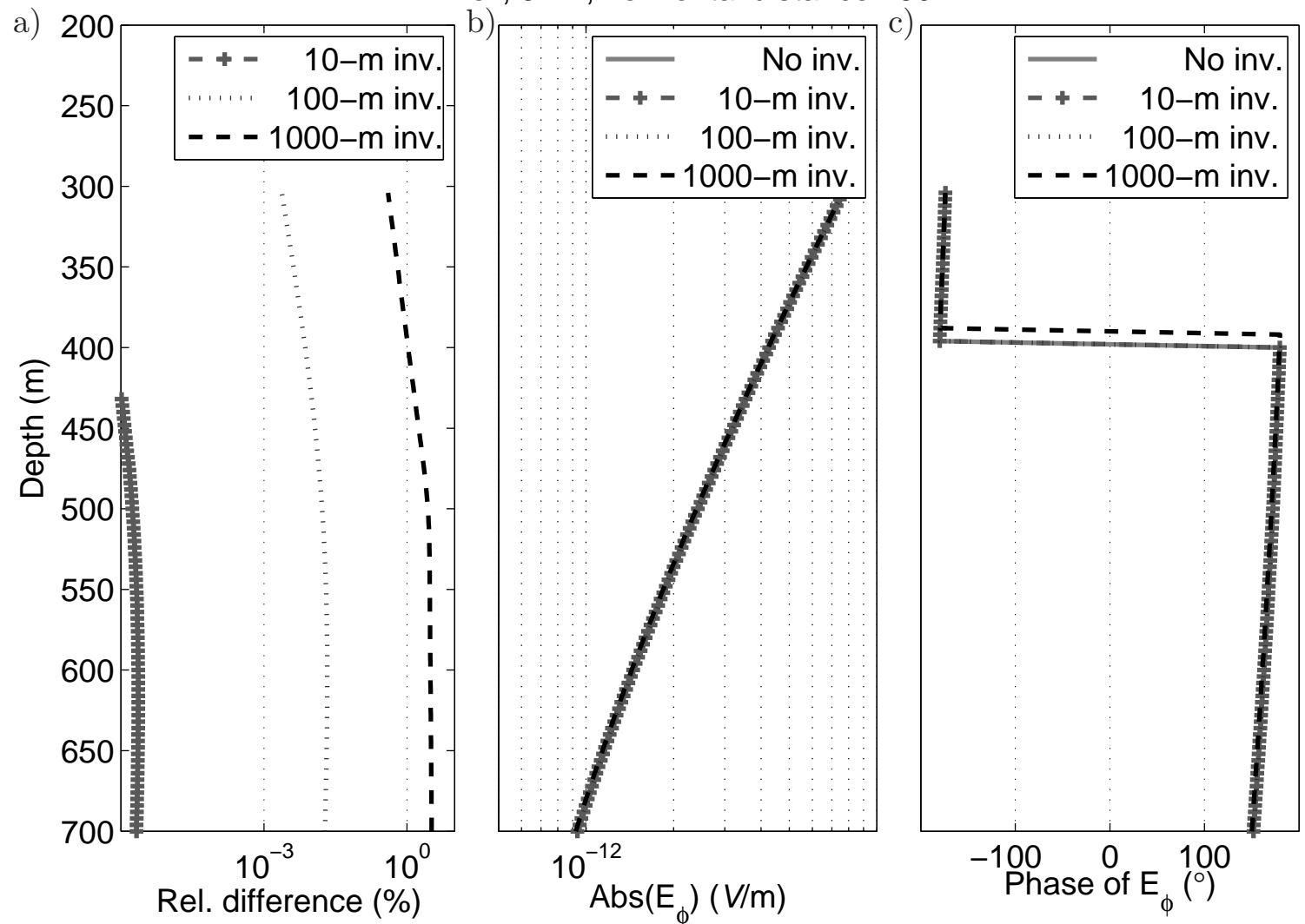

Figure 2: Cross well acquisition with a nearby well located $250 \mathrm{~m}$ from the cased well. Operating frequency is $5 \mathrm{~Hz}$. Different curves correspond to various radii of pistonlike water invasion. We display (a) the relative difference (in percent) with respect to the solution corresponding to the noninvaded formation and (b) the absolute value and (c) phase of the electric field.

larger than the one observed in Figure 2. In addition, if the receiver is located above the target layer, the sensitivity to water invasion exhibits only a slight decrease when the receiver well is located at a horizontal distance of $900 \mathrm{~m}$, as opposed to the sudden decrease observed in Figure 2.

\section{Cross-Well Measurements. $30 \mathrm{~Hz}, 900$ m (Horizontal Distance)}

Figure 5 displays EM measurements simulated in a nearby well located $900 \mathrm{~m}$ from the cased well at $30 \mathrm{~Hz}$. From the simulated measurements, we observe a sensitivity to radial extent of water invasion as high as $13 \%$ if the receiver is located below the target (oil-saturated) layer. In this case, if the receiver is located above the target layer, the sensitivity to water invasion rapidly decreases. The amplitude of the received signal is roughly one order of magnitude smaller than for the received EM signal simulated at $5 \mathrm{~Hz}$ (see Figure 4). 
X-well, $30 \mathrm{~Hz}$, horizontal distance $250 \mathrm{~m}$
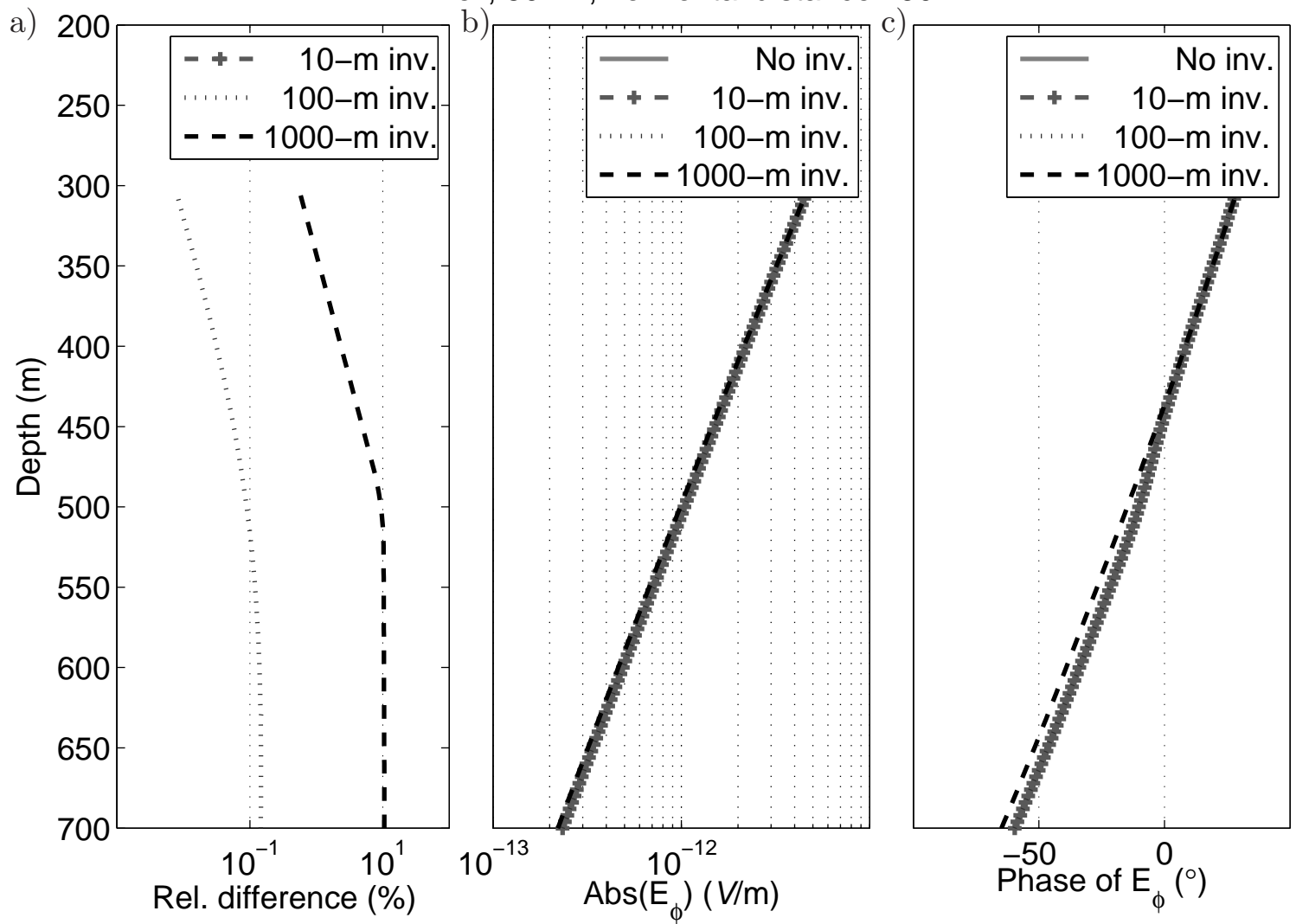

Figure 3: Cross well acquisition with a nearby well located $250 \mathrm{~m}$ from the cased well. Operating frequency is $30 \mathrm{~Hz}$. Different curves correspond to various radii of pistonlike water invasion. We display (a) the relative difference (in percent) with respect to the solution corresponding to the noninvaded formation and (b) the absolute value and (c) phase of the electric field.

\section{Variations of Resistivity in the Target (Oil-Saturated) Layer}

Figure 6 displays EM measurements simulated in a nearby well located $500 \mathrm{~m}$ from the cased well at $10 \mathrm{~Hz}$. We consider the case without water invasion. We observe a sensitivity to the resistivity of the target (oil-saturated) layer as high as $2 \%$ if the receiver is located below the target (oil-saturated) layer. For a receiver located above the target layer, the sensitivity of the measurements to the resistivity of the target layer rapidly decreases, and the intensity of the measured signal increases.

\section{Surface-to-Surface Measurements}

Figure 7 displays surface-to-surface measurements simulated at $10 \mathrm{~Hz}$. The sensitivity with respect to radial extent of water invasion increases to $1 \%$ as we consider larger horizontal distances between transmitter and receiver. We also observe a rapid decrease in the amplitude of the EM fields as we increase the horizontal distances between transmitter and receiver, as physically expected. For a pistonlike water invasion profile of radius equal to 
X-well, $5 \mathrm{~Hz}$, horizontal distance $900 \mathrm{~m}$
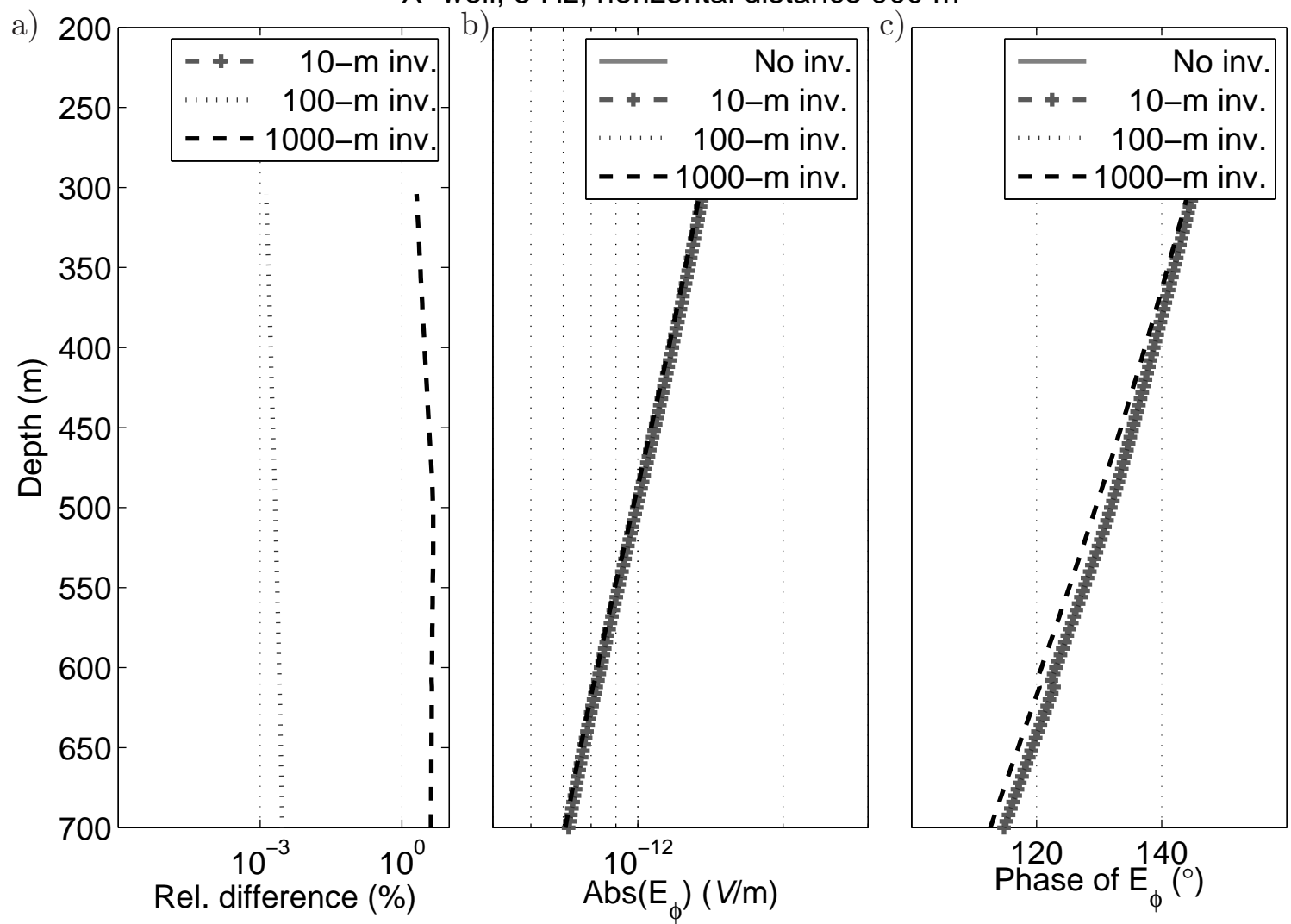

Figure 4: Cross well acquisition with a nearby well located $900 \mathrm{~m}$ from the cased well. Operating frequency is $5 \mathrm{~Hz}$. Different curves correspond to various radii of pistonlike water invasion. We display (a) the relative difference (in percent) with respect to the solution corresponding to the noninvaded formation and (b) the absolute value and (c) phase of the electric field.

or smaller than $100 \mathrm{~m}$, the sensitivity of the measurements decreases to a negligible level (below $0.001 \%$ ).

\section{Borehole-to-Surface Measurements}

Figure 8 displays borehole-to-surface EM measurements simulated at $10 \mathrm{~Hz}$. The transmitter is placed $600 \mathrm{~m}$ below surface, and the receiver $3 \mathrm{~m}$ below surface. In this case, we observe a sensitivity of the measurements with respect to radial extent of water invasion as large as 15\%, independently of the horizontal distance between transmitter and receiver. The largest amplitude of the simulated EM fields occurs when the horizontal distance between transmitter and receiver is within the range of $300-600 \mathrm{~m}$. 
X-well, $30 \mathrm{~Hz}$, horizontal distance $900 \mathrm{~m}$

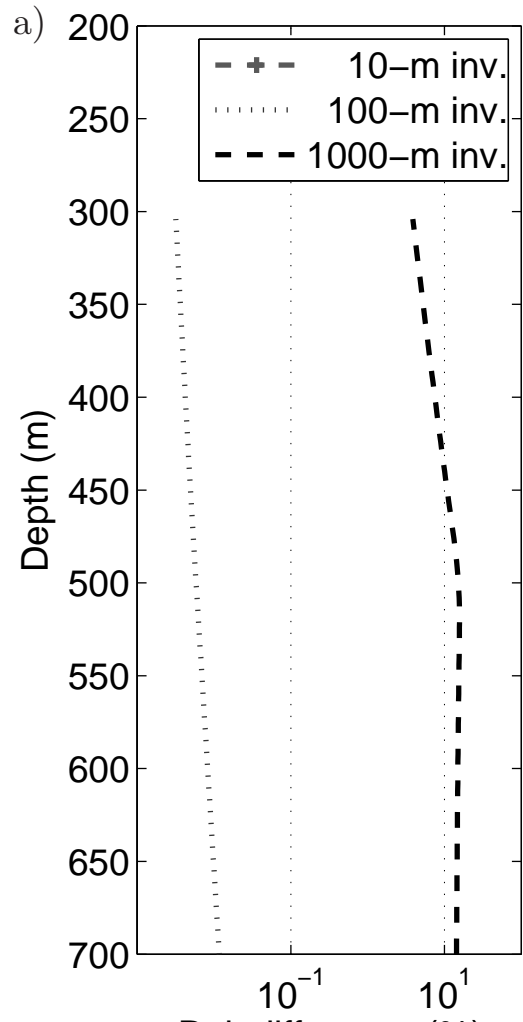

Rel. difference (\%)
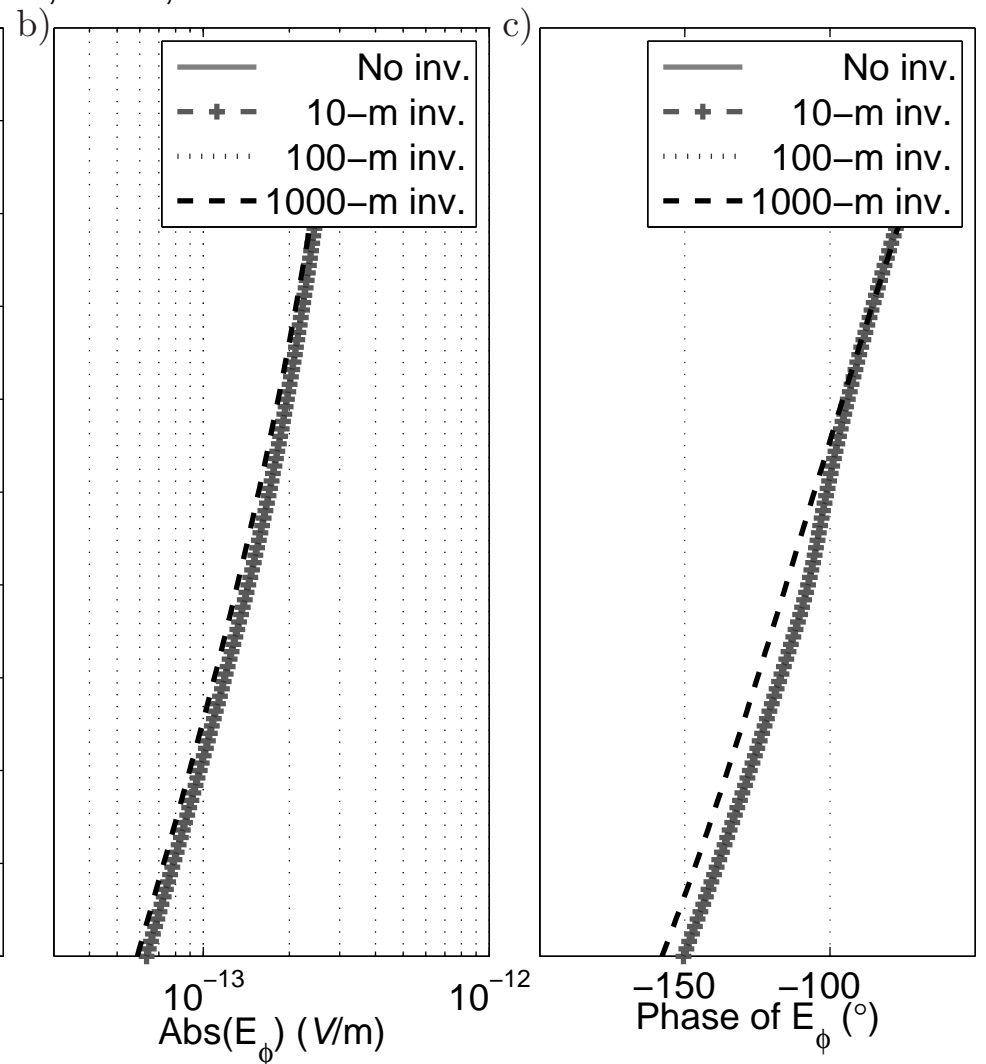

Figure 5: Cross well acquisition with a nearby well located $900 \mathrm{~m}$ from the cased well. Operating frequency is $30 \mathrm{~Hz}$. Different curves correspond to various radii of pistonlike water invasion. We display (a) the relative difference (in percent) with respect to the solution corresponding to the noninvaded formation and (b) the absolute value and (c) phase of the electric field.

\section{Surface-to-Borehole Measurements}

As shown in Figure 9, when placing the transmitter antenna $3 \mathrm{~m}$ below surface and the receiver antenna down-hole (600 $\mathrm{m}$ below surface), we observe similar results to those shown in Figure 8. In particular, we observe a measurement sensitivity with respect to radial extent of water invasion as large as $10 \%$, independently of the horizontal distance between transmitter and receiver. For this case, the largest amplitude of the simulated EM fields occurs when the horizontal distance between transmitter and receiver is within the range of $300-600 \mathrm{~m}$.

\section{Cross-Well Measurements}

Figure 10 describes cross-well measurements simulated at $10 \mathrm{~Hz}$. Both transmitter and receiver are placed $600 \mathrm{~m}$ below surface. We observe a sensitivity of the simulated measurements to radial extent of water invasion as large as $15 \%$, when the horizontal distance between both wells is above $300 \mathrm{~m}$. 
X-well, $10 \mathrm{~Hz}$, horizontal distance $500 \mathrm{~m}$
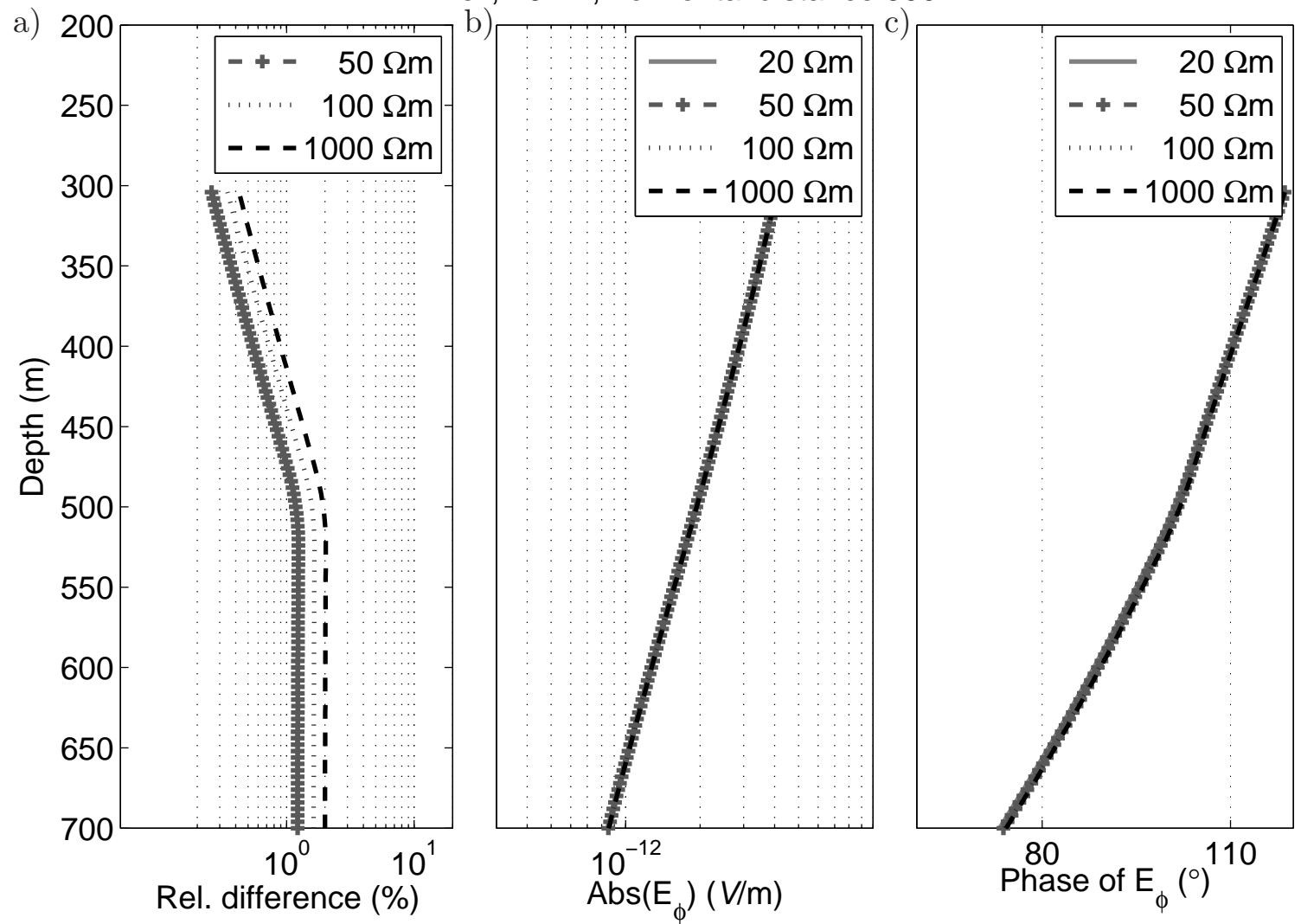

Figure 6: Cross well acquisition with a nearby well located $500 \mathrm{~m}$ from the cased well. Operating frequency is $10 \mathrm{~Hz}$. No water invasion occurred. Different curves correspond to various resistivities of the target (oil-saturated) layer. We display (a) the relative difference (in percent) with respect to the solution corresponding to the $20-\Omega \mathrm{m}$ resistive layer and (b) the absolute value and (c) phase of the electric field.

\section{Frequency Variations}

Figure 11 displays surface-to-surface EM measurements simulated with a horizontal distance between transmitter and receiver equal to $500 \mathrm{~m}$. The largest measurement sensitivity to radial extent of water invasion is observed in the frequency range from $5 \mathrm{~Hz}$ to $30 \mathrm{~Hz}$. Within this frequency range, we observe the largest amplitude of the EM fields simulated at the receiver antenna.

\section{Variations in Background Resistivity}

Figure 12 displays surface-to-surface EM measurements simulated at $10 \mathrm{~Hz}$ with a horizontal distance between transmitter and receiver equal to $500 \mathrm{~m}$. In this model, we vary the background conductivity. As we increase the background resistivity, we observe a larger measurement sensitivity to radial extent of water invasion, as well as a larger amplitude of the EM fields simulated at the receiver antenna. Once the background resistivity attains a value of $50 \Omega \mathrm{m}$, the sensitivity with respect to water invasion remains constant. 
X-well, $10 \mathrm{~Hz}$, Tx $3 \mathrm{~m}$ below surface, $\mathrm{Rx} 3 \mathrm{~m}$ below surface
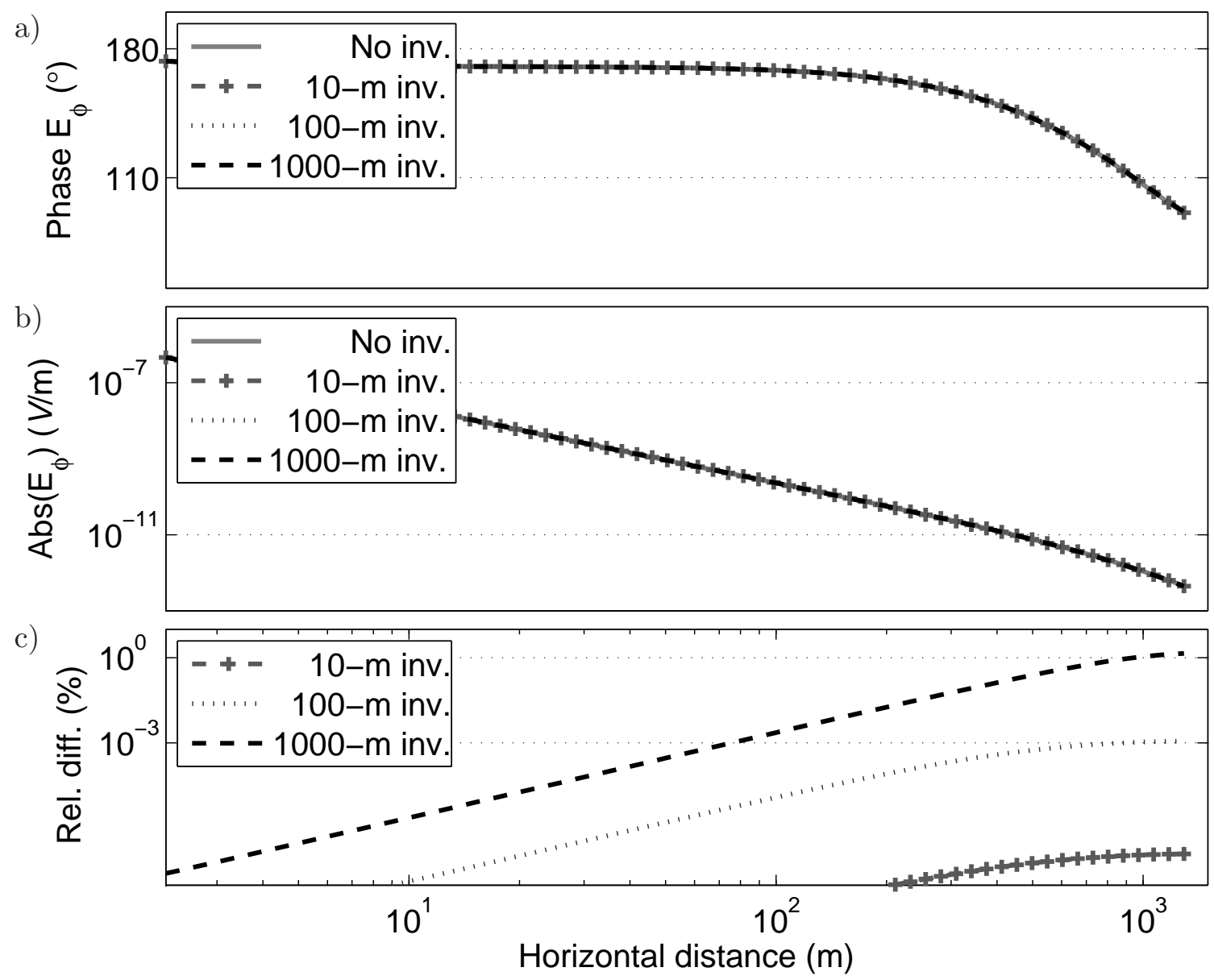

Figure 7: Surface-to-surface acquisition, with transmitter and receiver located $3 \mathrm{~m}$ below the surface. Operating frequency is $10 \mathrm{~Hz}$. Different curves correspond to various radii of pistonlike water invasion. We display (a) the phase and (b) absolute value of the electric field, and (c) the relative difference (in percent) with respect to the solution corresponding to the noninvaded formation.

\section{DISCUSSION}

Numerical results clearly indicate that EM measurements performed in the presence of casing are weakly sensitive to variations in the electrical and geometrical properties of rock formations. The largest simulated sensitivity with respect to water invasion was 15-20\% of the measured signal. This largest sensitivity to water invasion was attained when one antenna (transmitter or receiver) was located above the target (oil-saturated) layer, and the other antenna was located below the target layer.

The distance between transmitter and receiver governs the sensitivity and range of amplitude variations of the simulated EM fields. Simulation results indicate that, in most cases, the amplitude range of the electric field anomaly increases as the distance between source and receiver increases.

For the subsurface conductivity model and casing properties considered in this work, 
X-well, $10 \mathrm{~Hz}$, Tx 600 m below surface, Rx 3 m below surface
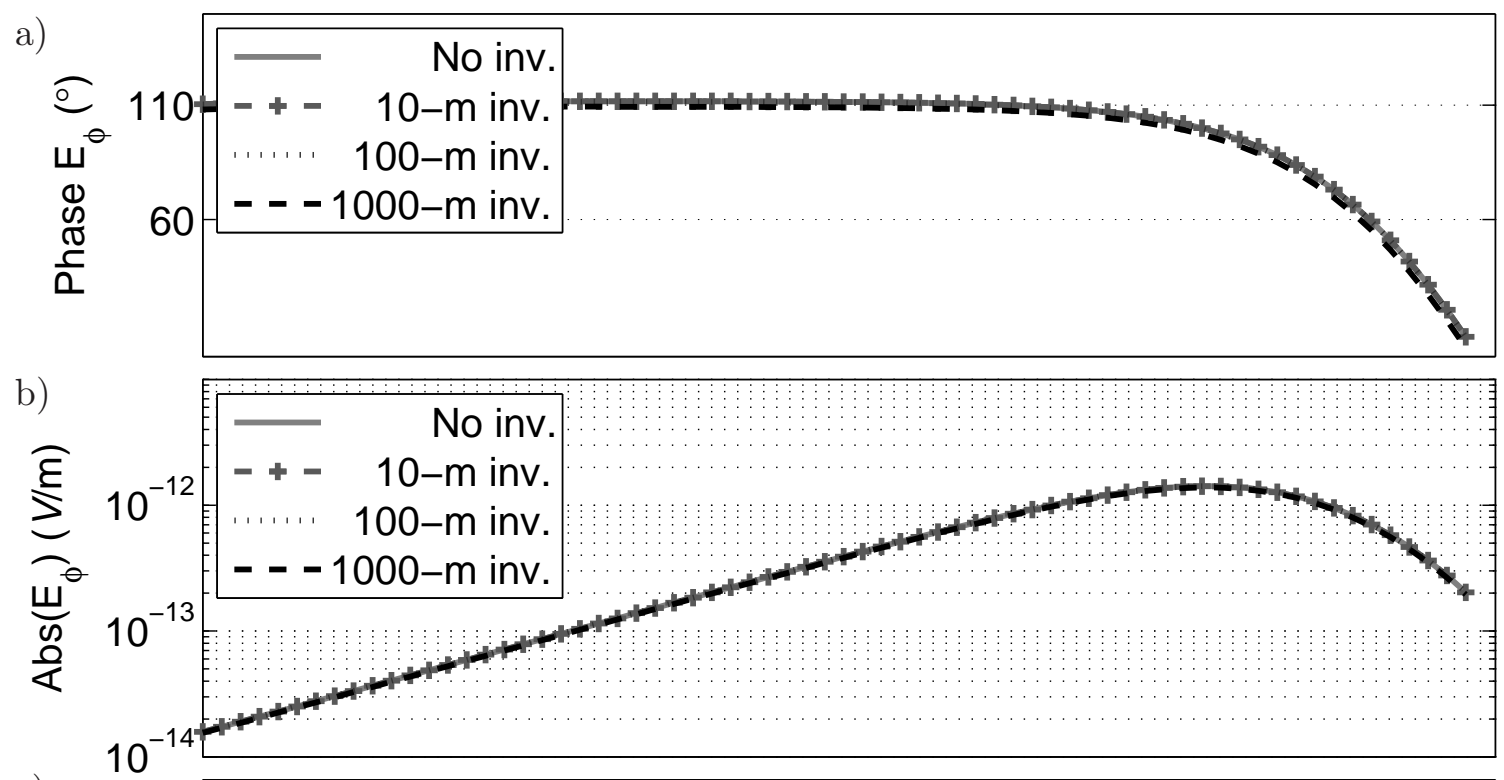

c)

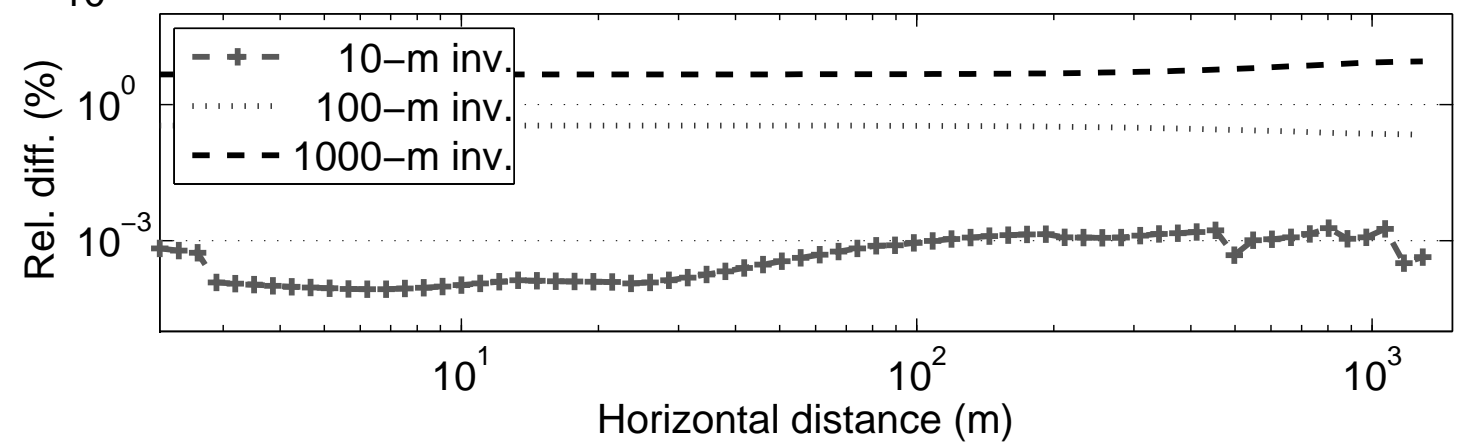

Figure 8: Borehole-to-surface acquisition, with transmitter located $600 \mathrm{~m}$ below the surface, and receiver located $3 \mathrm{~m}$ below the surface. Operating frequency is $10 \mathrm{~Hz}$. Different curves correspond to various radii of pistonlike water invasion. We display (a) the phase and (b) absolute value of the electric field and (c) the relative difference (in percent) with respect to the solution corresponding to the noninvaded formation.

numerical simulations indicate that the range of variation of the electric field is between $10^{-14}$ and $10^{-7} \mathrm{~V} / \mathrm{m}$ for a frequency range between 5 and $30 \mathrm{~Hz}$. Such ranges of variations would be difficult to simulate with a relative accuracy better than $1 \%$ with a finite-difference code. The self-adaptive goal-oriented $h p$ method is not only capable of achieving this numerical accuracy but also allows one to quantify the effect of small variations in the geometrical and physical properties of both casing and cement.

\section{CONCLUSIONS}

The following conclusions stem from the numerical simulation results described in this work.

The frequency range from $5 \mathrm{~Hz}$ to $30 \mathrm{~Hz}$ provides optimal results in the sense that it delivers both maximum measurement sensitivity with respect to water invasion and maximum 
X-well, $10 \mathrm{~Hz}$, Tx 3 m below surface, Rx 600 m below surface
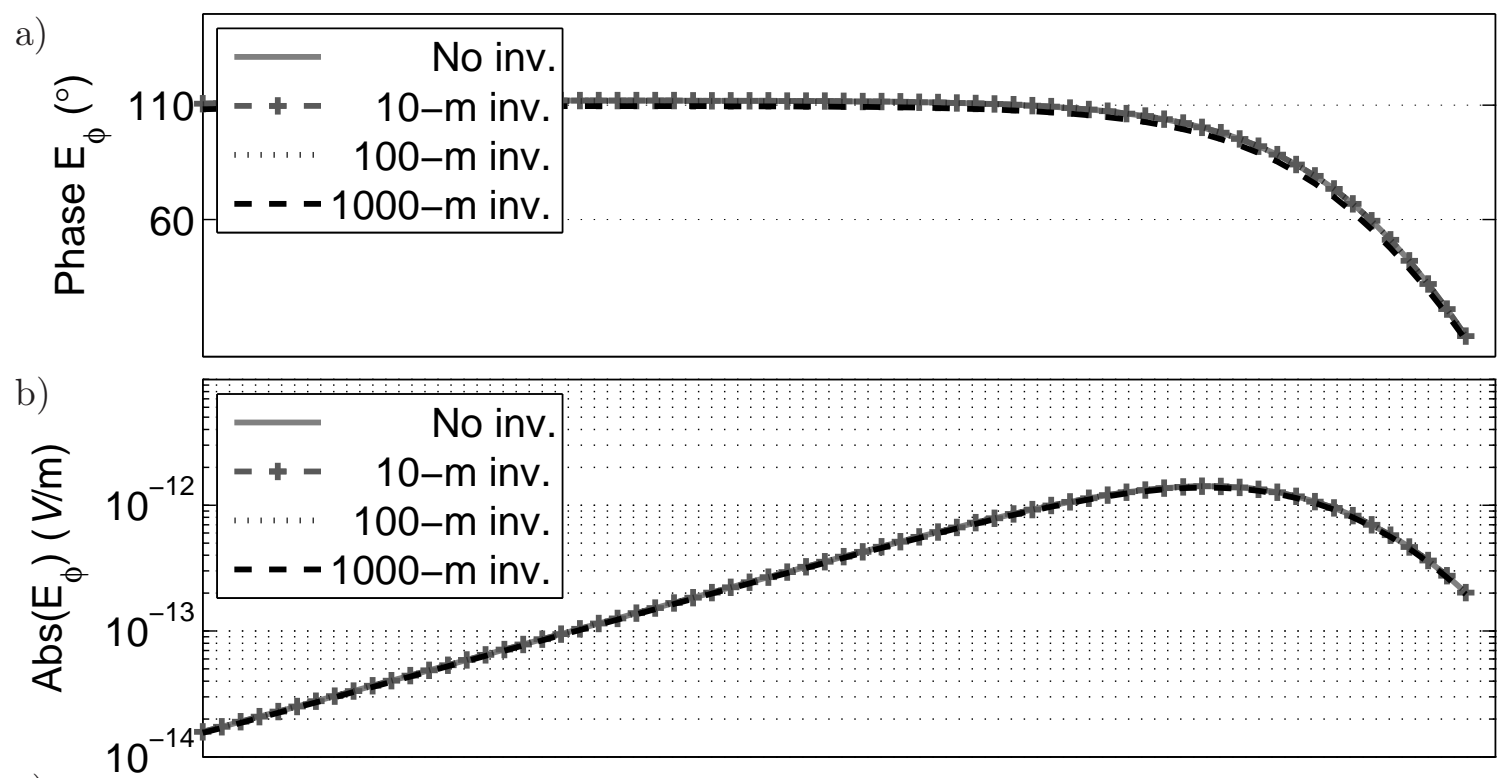

c)

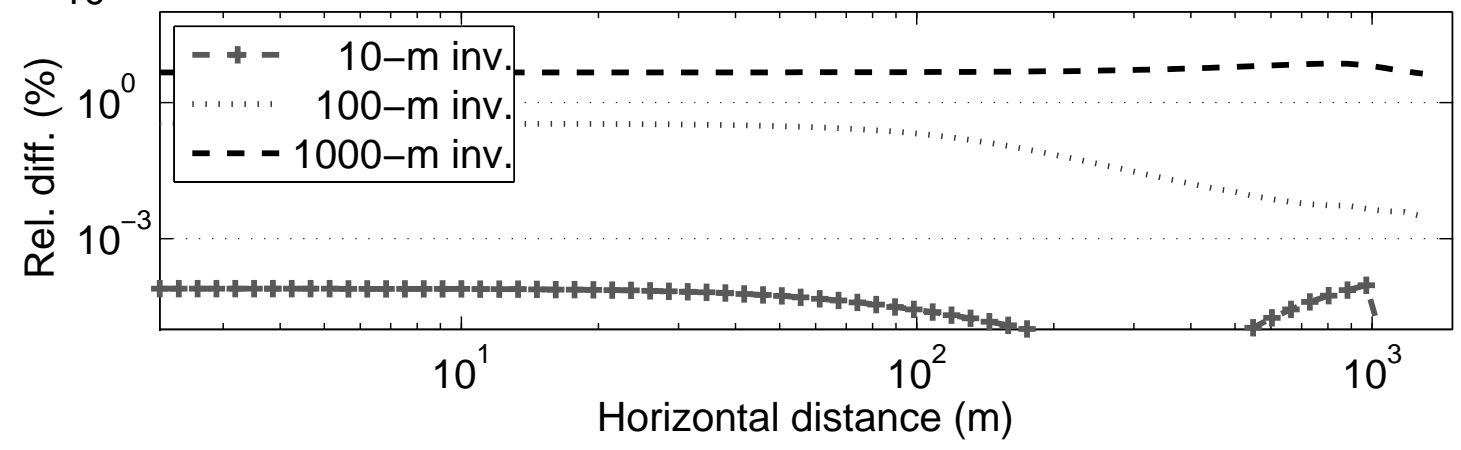

Figure 9: Surface-to-borehole acquisition, with transmitter located $3 \mathrm{~m}$ below the surface and receiver located $600 \mathrm{~m}$ below the surface. Operating frequency is $10 \mathrm{~Hz}$. Different curves correspond to various radii of pistonlike water invasion. We display (a) the phase and (b) absolute value of the electric field and (c) the relative difference (in percent) with respect to the solution corresponding to the noninvaded formation.

strength of the simulated EM fields at the receiver antenna. Therefore, such a frequency range appears to be the most adequate for field acquisition.

Simulated measurements are more sensitive to water invasion effects when the formation resistivity is above $50 \Omega \mathrm{m}$, indicating that to monitor water invasion using surface-to-surface measurements should not be pursued in highly conductive formations.

The combined use of a down-hole antenna (below the target layer) and another antenna close to the surface (above the target layer) enhances the capabilities of identifying and estimating the thickness of the target layer and the radius of water invasion. 
X-well, $10 \mathrm{~Hz}$, Tx $600 \mathrm{~m}$ below surface, $\mathrm{Rx} 600 \mathrm{~m}$ below surface

a)
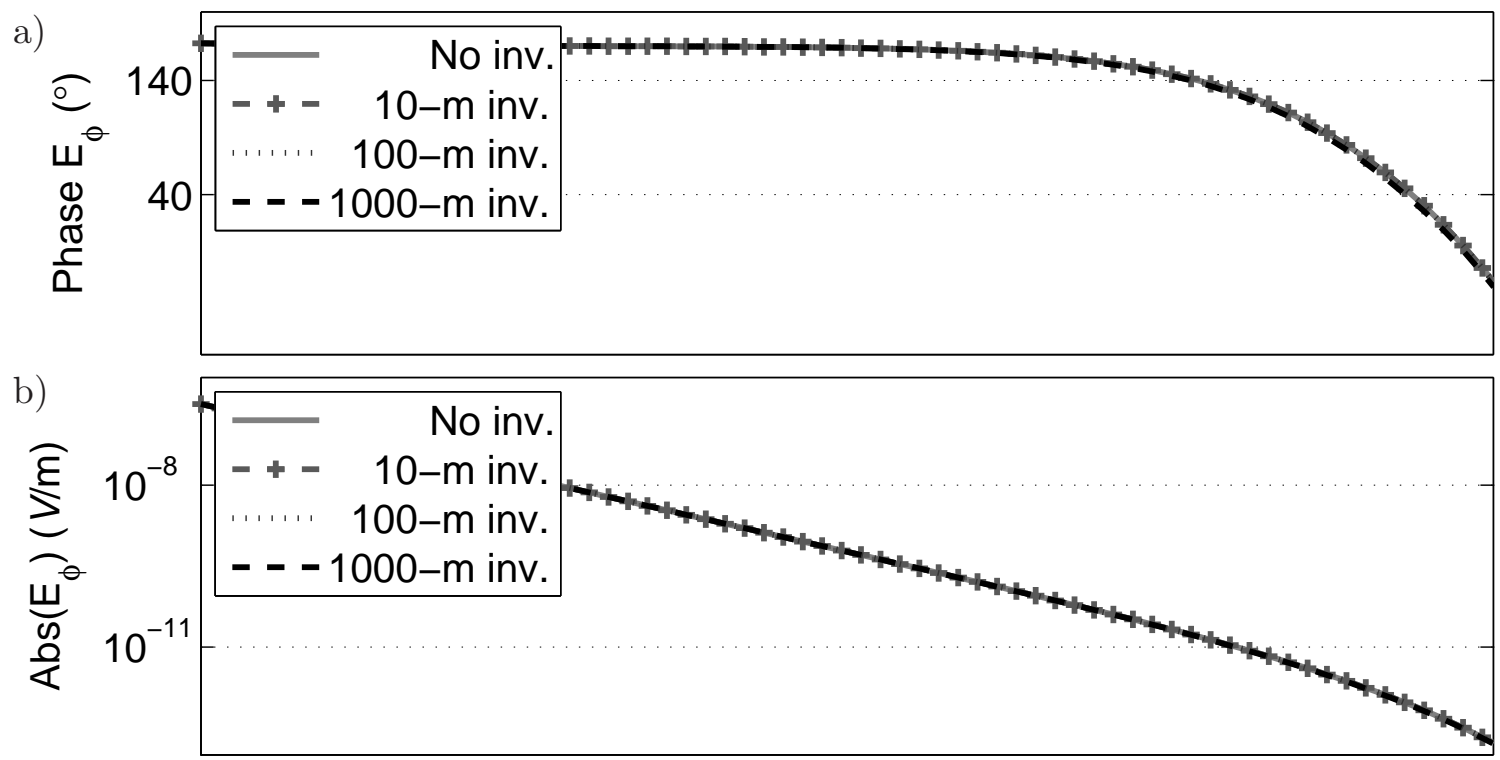

c)

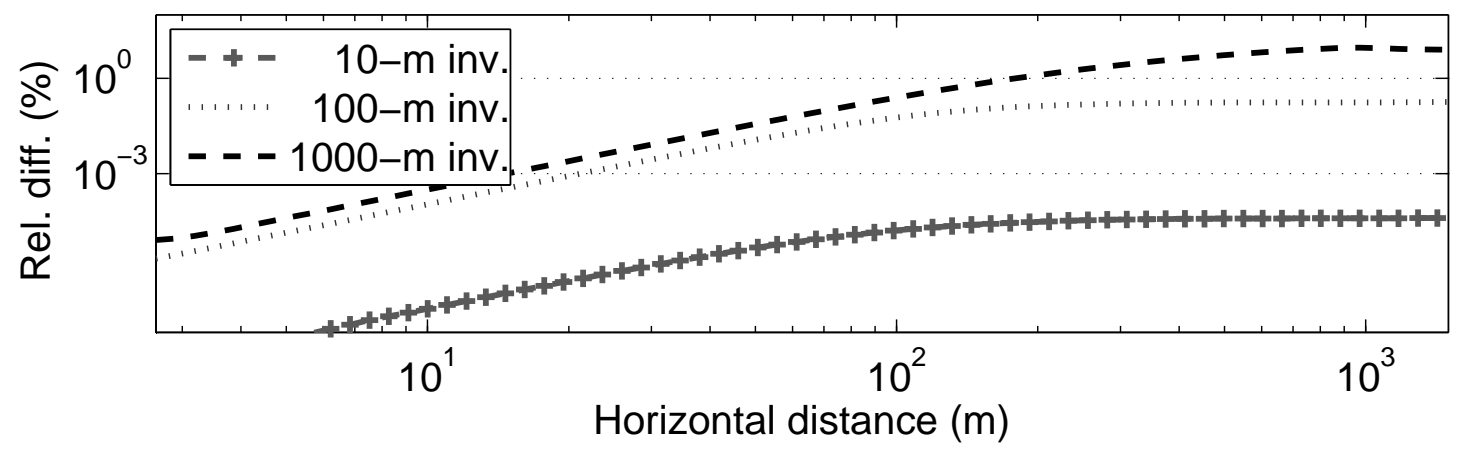

Figure 10: Cross well configuration, with transmitter and receiver located $600 \mathrm{~m}$ below the surface. Operating frequency is $10 \mathrm{~Hz}$. Different curves correspond to various radii of pistonlike water invasion. We display (a) the phase and (b) absolute value of the electric field, and (c) the relative difference (in percent) with respect to the solution corresponding to the noninvaded formation.

\section{ACKNOWLEDGMENTS}

The authors thank Shell International E\&P for funding of the work and for permission to publish this paper.

\section{APPENDIX}

\section{FORMULATION}

We describe the variational formulation used to solve the azimuthal component of the EM fields in an axisymmetric formation. Using cylindrical coordinates $(\rho, \phi, z)$, the variational formulation in terms of the azimuthal component of the electric field $\mathbf{E}_{\phi}=\left(0, E_{\phi}, 0\right)$ is 
a) Horizontal distance $500 \mathrm{~m}$, Tx and Rx 3 m below surface

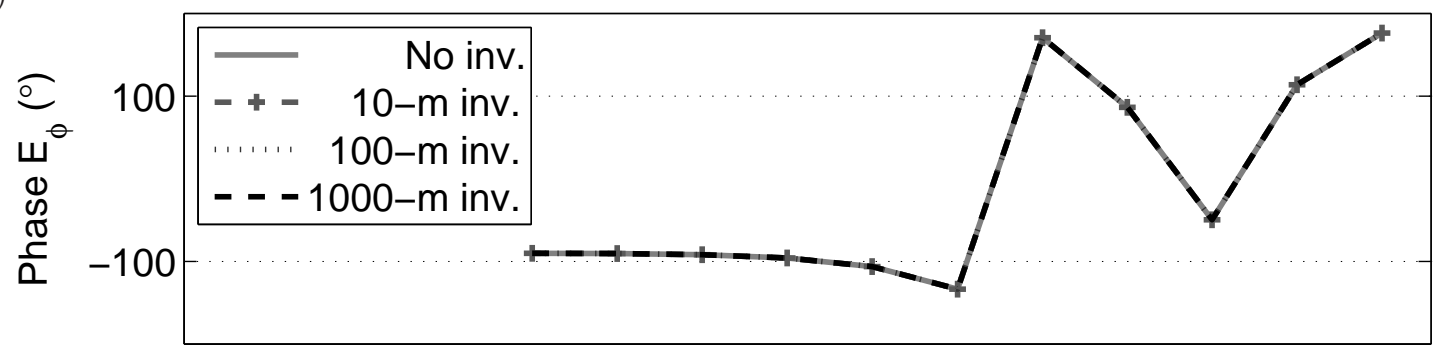

b)

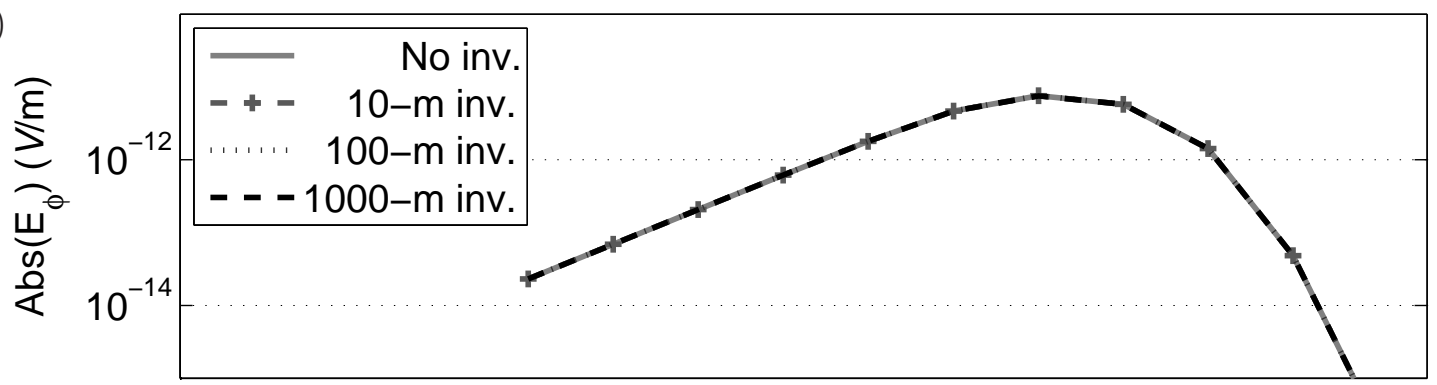

c)

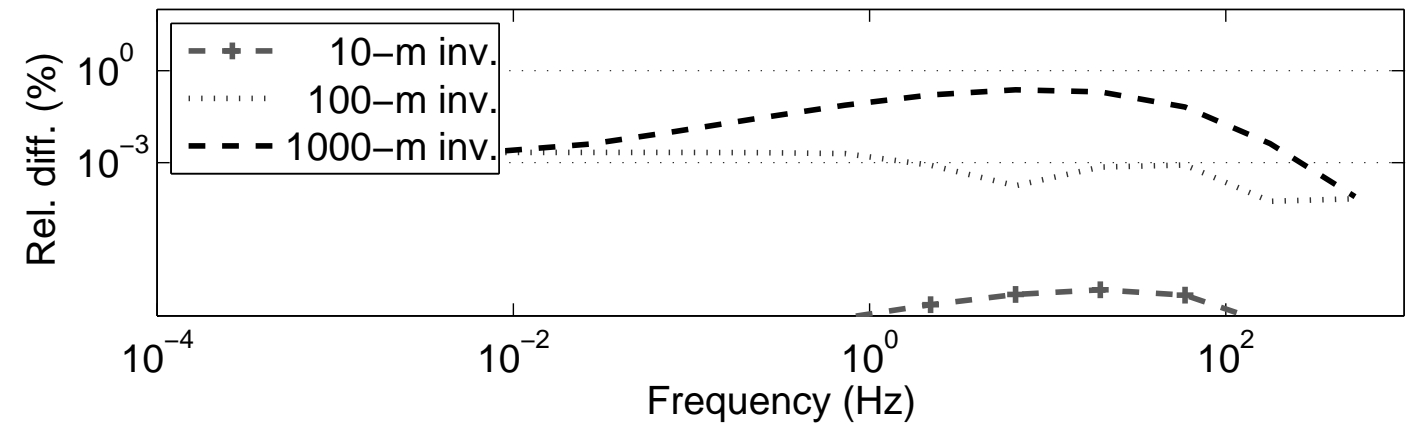

Figure 11: Surface-to-surface acquisition with a nearby well located $500 \mathrm{~m}$ from the cased well. Different curves correspond to various radii of pistonlike water invasion. We display (a) the phase and (b) absolute value of the electric field and (c) the relative difference (in percent) with respect to the solution corresponding to the noninvaded formation as a function of the frequency.

given by:

$$
\left\{\begin{array}{l}
\text { Find } E_{\phi} \in E_{\phi, D}+\tilde{H}_{D}^{1}(\Upsilon) \text { such that: } \\
\int_{\Upsilon}\left(\overline{\overline{\boldsymbol{\mu}}}_{\rho, z}^{-1} \boldsymbol{\nabla} \times \mathbf{E}_{\phi}\right) \cdot\left(\boldsymbol{\nabla} \times \overline{\mathbf{F}}_{\phi}\right) d V \\
-\int_{\Upsilon}\left[\left(\omega^{2} \overline{\overline{\boldsymbol{\epsilon}}}_{\phi}-j \omega \overline{\overline{\boldsymbol{\sigma}}}_{\phi}\right) \mathbf{E}_{\phi}\right] \cdot \overline{\mathbf{F}}_{\phi} d V \\
=-j \omega \int_{\Upsilon} J_{\phi}^{i m p} \bar{F}_{\phi} d V+j \omega \int_{\Gamma_{N}} J_{\phi, \Gamma_{N}}^{i m p} \bar{F}_{\phi} d S \\
-\int_{\Upsilon}\left(\overline{\overline{\boldsymbol{\mu}}}_{\rho, z}^{-1} \mathbf{M}_{\rho, z}^{i m p}\right) \cdot \nabla \times \overline{\mathbf{F}}_{\phi} d V \quad \forall F_{\phi} \in \tilde{H}_{D}^{1}(\Upsilon),
\end{array}\right.
$$

where $j=\sqrt{-1}$ is the imaginary unit, $\omega$ is radian (angular) frequency, $\Upsilon$ is the computational domain, $E_{\phi, D}$ represents the Dirichlet data (typically $E_{\phi, D}=0$ ), $\overline{\overline{\boldsymbol{\sigma}}}_{\phi}, \overline{\overline{\boldsymbol{\epsilon}}}_{\phi}$ are the azimuthal components of the conductivity and permittivity tensors, respectively, $\overline{\overline{\boldsymbol{\mu}}}_{\rho, z}$ is the meridian component of the magnetic permeability tensor, $J_{\phi}^{i m p}$ is the azimuthal compo- 

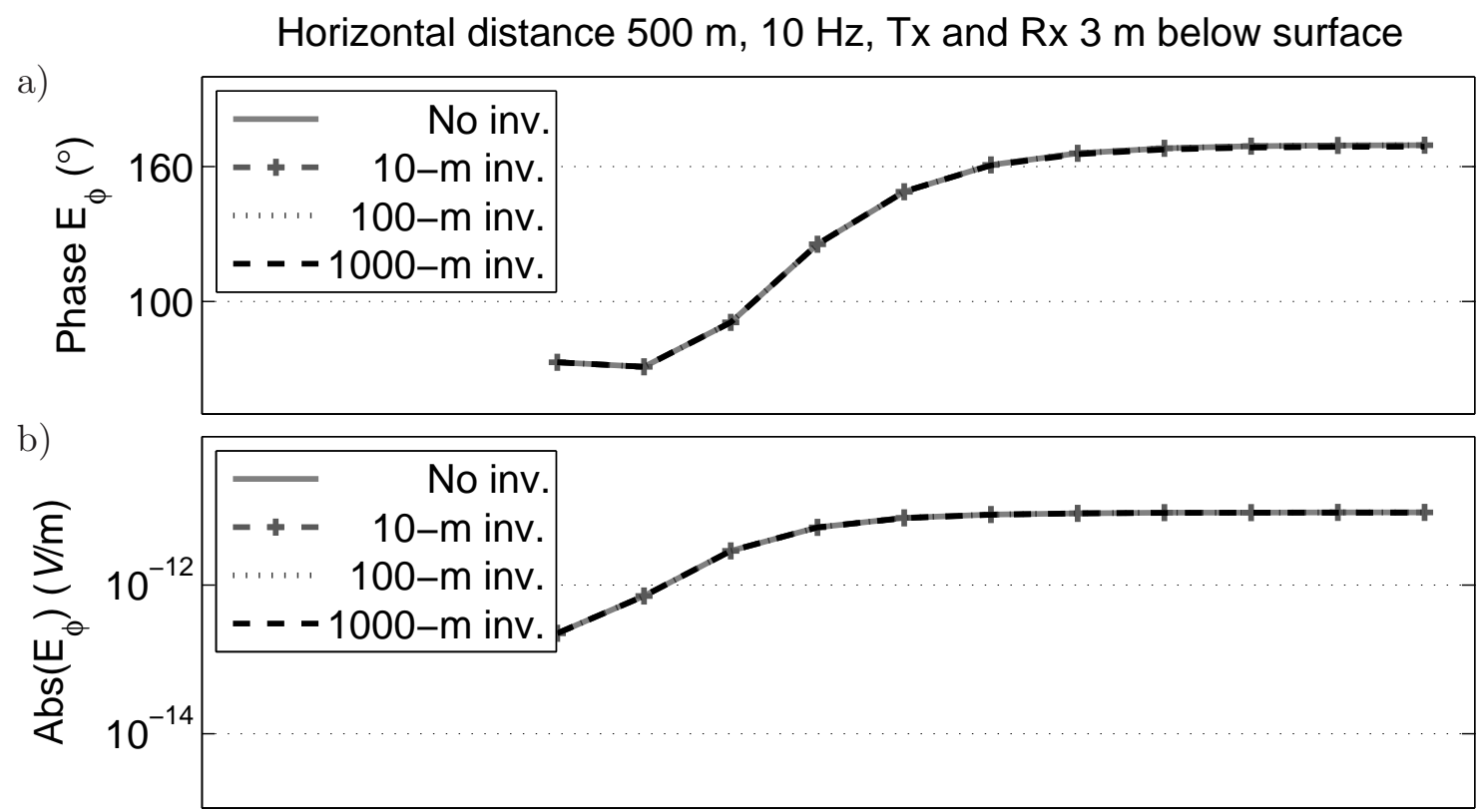

c)

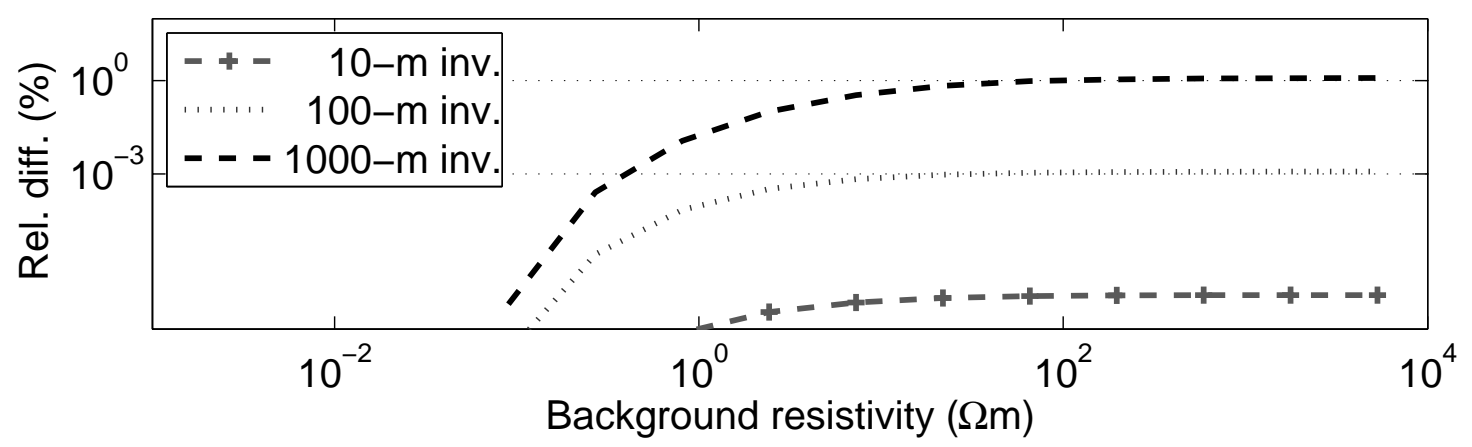

Figure 12: Surface-to-surface acquisition with a nearby well located $500 \mathrm{~m}$ from the cased well at $10 \mathrm{~Hz}$. Different curves correspond to various radii of pistonlike water invasion. We display (a) the phase and (b) absolute value of the electric field and (c) the relative difference (in percent) with respect to the solution corresponding to the noninvaded formation as a function of the background resistivity.

nent of the impressed electric current, $\mathbf{M}_{\rho, z}^{i m p}$ is the meridian component of the impressed magnetic current, $\Gamma_{D}$ and $\Gamma_{N}$ are the segments of the boundary where we impose Dirichlet (essential) and Neumann (natural) boundary conditions, respectively, $\mathbf{F}_{\phi}=\left(0, F_{\phi}, 0\right)$ is a test function with $\overline{\mathbf{F}}_{\phi}=\left(0, \bar{F}_{\phi}, 0\right)$ being its complex conjugate, and $F_{\phi} \in \tilde{H}_{D}^{1}(\Upsilon)=\left\{E_{\phi}\right.$ : $\left.\left(0, E_{\phi}, 0\right) \in H_{D}(\operatorname{curl} ; \Upsilon)\right\}=\left\{E_{\phi} \in L^{2}(\Upsilon): \frac{1}{\rho} E_{\phi}+\frac{\partial E_{\phi}}{\partial \rho} \in L^{2}(\Upsilon), \frac{\partial E_{\phi}}{\partial z} \in L^{2}(\Upsilon),\left.E_{\phi}\right|_{\Gamma_{D}}=0\right\}$ For a detailed derivation of the above formulation, see Pardo et al. (2007a).

The corresponding dual variational formulation in terms of the azimuthal component of 
the magnetic field $\mathbf{H}_{\phi}=\left(0, H_{\phi}, 0\right)$ is given by:

$$
\left\{\begin{array}{l}
\text { Find } H_{\phi} \in H_{\phi, D}+\tilde{H}_{D}^{1}(\Upsilon) \text { such that: } \\
\int_{\Upsilon}\left[\left(\overline{\overline{\boldsymbol{\sigma}}}_{\rho, z}+j \omega \overline{\overline{\boldsymbol{\epsilon}}}_{\rho, z}\right)^{-1} \nabla \times \mathbf{H}_{\phi}\right] \cdot\left(\boldsymbol{\nabla} \times \overline{\mathbf{F}}_{\phi}\right) d V \\
+j \omega \int_{\Upsilon}\left(\overline{\overline{\boldsymbol{\mu}}}_{\phi} \mathbf{H}_{\phi}\right) \cdot \overline{\mathbf{F}}_{\phi} d V= \\
-\int_{\Upsilon} M_{\phi}^{i m p} \bar{F}_{\phi} d V+\int_{\Gamma} M_{\phi, \Gamma}^{i m p} \bar{F}_{\phi} d S \\
+\int_{\Upsilon}\left[\left(\overline{\overline{\boldsymbol{\sigma}}}_{\rho, z}+j \omega \overline{\overline{\boldsymbol{\epsilon}}}_{\rho, z}\right)^{-1} \mathbf{J}_{\rho, z}^{i m p}\right] \cdot \nabla \times \overline{\mathbf{F}}_{\phi} d V \\
\forall F_{\phi} \in \tilde{H}_{D}^{1}(\Upsilon),
\end{array}\right.
$$

where $H_{\phi, D}$ represents the Dirichlet data (typically $H_{\phi, D}=0$ ), $\overline{\overline{\boldsymbol{\sigma}}}_{\rho, z}, \overline{\overline{\boldsymbol{\epsilon}}}_{\rho, z}$ are the meridian components of the conductivity and permittivity tensors, respectively, $\overline{\overline{\boldsymbol{\mu}}}_{\rho, z}$ is the azimuthal component of the magnetic permeability tensor, $M_{\phi}^{i m p}$ is the azimuthal component of the impressed magnetic current, and $\mathbf{J}_{\rho, z}^{i m p}$ is the meridian component of the impressed electric current. For a detailed derivation of the above formulation, see Pardo et al. (2007a).

\section{REFERENCES}

Augustin, A. M., W. D. Kennedy, H. F. Morrison, and K. H. Lee, 1989, A theoretical study of surface-to-borehole electromagnetic logging in cased holes: Geophysics, 54, 90-99.

Bevc, D., and H. F. Morrison, 1991, Borehole-to-surface electrical resistivity monitoring of a salt water injection experiment: Geophysics, 56, 769-777.

Demkowicz, L., 2006, Computing with hp-adaptive finite elements, v. 1: One and two dimensional elliptic and Maxwell problems: Chapman and Hall.

Hoversten, G. M., P. Milligan, J. Byun, J. Washbourne, L. C. Knauer, and P. Harness, 1995, Crosswell electromagnetic and seismic imaging: An examination of coincident surveys at a steam flood project: Geophysics, 60, 912-920.

Kirkendall, B., P. Harben, and P. Lewis, 1999, Advances in crosswell electromagnetics: Steel cased boreholes: SEG Expanded Abstracts, 18, 323-326.

Nekut, A. G., 1995, Crosswell electromagnetic tomography in steel-cased wells: Geophysics, 60, no. 3, 912-920.

Newmark, R. L., W. Daily, and A. Ramirez, 1999, Electrical resistance tomography using steel cased borehole as electrodes: SEG Expanded Abstracts, 18, 327-330.

Pardo, D., L. Demkowicz, C. Torres-Verdín, and M. Paszynski, 2006a, Simulation of resistivity logging-while-drilling (LWD) measurements using a self-adaptive goal-oriented hp-finite element method: SIAM Journal on Applied Mathematics, 66, no. 6, 2085-2106.

- 2007a, A goal oriented hp-adaptive finite element strategy with electromagnetic applications. Part 2: Electrodynamics: Computer Methods in Applied Mechanics and Engineering., 196, 3585-3597.

Pardo, D., L. Demkowicz, C. Torres-Verdín, and L. Tabarovsky, 2006b, A goal-oriented hpadaptive finite element method with electromagnetic applications. Part 1: Electrostatics: International Journal for Numerical Methods in Engineering, 65, no. 8, 1269-1309.

Pardo, D., C. Torres-Verdín, and L. Demkowicz, 2006c, Simulation of multi-frequency borehole resistivity measurements through metal casing using a goal-oriented $h p$-finite element method: IEEE Transactions on Geosciences and Remote Sensing, 44, no. 8, 2125-2135. 
— $2007 \mathrm{~b}$, Feasibility study for two-dimensional frequency dependent electromagnetic sensing through casing: Geophysics, 72, no. 3, F111-F118.

Paszynski, M., L. Demkowicz, and D. Pardo, 2005, Verification of goal-oriented $h p$ adaptivity: Computers and Mathematics with Applications, 50, no. 8-9, 1395-1404.

Sasaki, Y., K. Matsuo, and K. Yokoi, 1994, Resistivity inversion of cross-hole and boreholeto-surface EM data using axially symmetric models: Geophysical Prospecting, 42, no. 7, $745-754$.

Spies, B. R., and T. M. Habashy, 1995, Sensitivity analysis of crosswell electromagnetics: Geophysics, 60, 834-845. 